Article

\title{
Trends in Antimicrobial Drug Resistance of Streptococcus pneumoniae Isolates at Jordan University Hospital (2000-2018)
}

\author{
Malik Sallam ${ }^{1,2,3, *}$, Jumana Abbadi ${ }^{1,2}$, Anas Natsheh ${ }^{1,2}$, Nidaa A. Ababneh ${ }^{4}$, \\ Azmi Mahafzah 1,2 and Gülşen Özkaya Şahin ${ }^{3,5}$ \\ 1 Department of Pathology, Microbiology and Forensic Medicine, School of Medicine, \\ The University of Jordan, Amman 19328, Jordan; jmb_abbadi@hotmail.com (J.A.); \\ natshehanas@gmail.com (A.N.); mahafzaa@gmail.com (A.M.) \\ 2 Department of Clinical Laboratories and Forensic Medicine, Jordan University Hospital, \\ Amman 22362, Jordan \\ 3 Department of Translational Medicine, Faculty of Medicine, Lund University, 22362 Malmö, Sweden; \\ gulsen.ozkaya_sahin@med.lu.se \\ 4 Cell Therapy Center (CTC), The University of Jordan, Amman 19328, Jordan; nidaaanwar@gmail.com \\ 5 Department of Clinical Microbiology, Laboratory Medicine, Skåne University Hospital, 22362 Lund, Sweden \\ * Correspondence: malik.sallam@ju.edu.jo; Tel.: +962-79-184-5186
}

Received: 11 March 2019; Accepted: 10 April 2019; Published: 12 April 2019

check for updates

\begin{abstract}
Antimicrobial drug resistance (AMR) in pneumococci complicates the treatment of serious pneumococcal infections. Country-specific AMR patterns can help to establish guidelines for empiric therapy. The aim of the current study was to analyze the distribution of AMR among Streptococcus pneumoniae isolates at Jordan University Hospital (JUH) during 2000-2018. Paper-based and electronic clinical data registry records from 2000 to 2018 were retrospectively analyzed to study the AMR among pneumococcal isolates at JUH. Temporal trend analysis was done using two-tailed linear-by-linear test for association. The total number of unique pneumococcal isolates that were identified was 556, of which 544 isolates had antimicrobial susceptibility testing results. The most frequent specimens were eye $(n=117,21.0 \%)$, bloodstream $(n=93,16.7 \%)$ and sputum $(n=81,14.6 \%)$. Invasive infections represented $23.6 \%$ of all unique isolates. The overall susceptibility of $S$. pneumoniae isolates during the study period to different antimicrobials was: $100 \%$ to vancomycin, $97.7 \%$ to ceftriaxone, $97.1 \%$ to cefotaxime, $94.9 \%$ to chloramphenicol, $89.7 \%$ to penicillin, $83.8 \%$ to levofloxacin, $67.7 \%$ to clindamycin and $52.1 \%$ to erythromycin. The prevalence of multi-drug resistance (MDR) was $8.6 \%$ (95\% confidence interval: $6.4-11.5 \%$ ). Trend analysis showed an increase in the prevalence of non-susceptibility to erythromycin, clindamycin and levofloxacin $(p<0.001)$. MDR prevalence increased from $1.6 \%$ in the first quarter to $14.6 \%$ in the fourth quarter $(p<0.001)$. The incidence of invasive infections declined over the study period $(p<0.001)$. The increase in the prevalence of AMR and MDR among pneumococcal isolates in Jordan demands judicious use of antimicrobials and regular surveillance of resistance.
\end{abstract}

Keywords: Streptococcus pneumoniae; meningitis; bacteremia; antibiotic; Middle East; Jordan; resistance; trend

\section{Introduction}

Streptococcus pneumoniae is a frequent colonizer of the human upper respiratory tract [1,2]. It is the causative agent for a wide variety of life-threatening infections including pneumonia, meningitis 
and bacteremia [3-5]. In addition, S. pneumoniae is a leading cause of otitis, sinusitis and bronchitis, particularly in children [6].

Beta-lactam antibiotics (e.g., penicillin, third-generation cephalosporins) are considered the mainstay of treatment for S. pneumoniae infections [7,8]. Macrolides (e.g., erythromycin), fluoroquinolones (e.g., levofloxacin), lincosamides (e.g., clindamycin) and vancomycin can be used for management of beta-lactam non-susceptible pneumococcal isolates and for individuals who cannot tolerate beta-lactams [8].

The emergence of antimicrobial drug resistance (AMR) in S. pneumoniae can hinder the management of pneumococcal infections [9]. Multi-drug resistance (MDR) among pneumococci is particularly a serious problem which is defined as resistance of an isolate to three or more classes of antibiotics [10-12]. The increasing prevalence of AMR among pneumococci poses serious therapeutic problems, mainly in the management of pneumococcal meningitis [13].

The investigation of AMR trends among pneumococcal isolates can help to understand the role of antibiotic therapy practices in the emergence of resistance and to revise the empiric therapy guidelines for pneumococcal disease at a country and in regional levels [11,14-16]. A number of factors have been postulated as the underlying causes behind the emergence of AMR, including: the wide availability of antibiotics as over-the-counter drugs in some countries, unnecessary prescriptions by physicians and non-compliance by the patients [17].

An increasing trend in the prevalence of penicillin-non-susceptible pneumococci have been reported in various regions globally [11,18-20]. In Jordan, a few reports indicated a similar pattern of an increasing AMR trend among pneumococcal isolates [21-24]. However, an updated report from the country is needed as the results of the previous reports are either outdated or focused on specific patient groups (e.g., children), in addition to the issues of small sample sizes and short study periods. Thus, the aim of this project was to study the prevalence and temporal trends of AMR among pneumococcal isolates at Jordan University Hospital (JUH) over a period of 19 years using data on all available isolates.

\section{Methods}

\subsection{Study Design}

This retrospective study was carried out using patient data that were collected from January 2000 to August 2018 at Jordan University Hospital (JUH), an academic teaching hospital in Amman, Jordan. JUH has a bed capacity of 550 and annually serves more than 500,000 patients in the outpatient clinics as of January 2019. Manual search (January 2000 till December 2008) and electronic search (January 2009 till August 2018) in the clinical data registry records were conducted by three authors (MS, JA and AN).

\subsection{Ethical Permission}

The study was approved by the Jordan University Hospital ethical review board (IRB/262/2018) in accordance with the declaration of Helsinki.

\subsection{Identification of S. pneumoniae Isolates and Antimicrobial Susceptibility Testing (AST)}

The presumptive identification of S. pneumoniae isolates at JUH depends on colonial morphology, alpha-hemolysis on $5 \%$ sheep blood agar (SBA) and sensitivity to optochin $(5 \mu \mathrm{g})$ following a 24-hour incubation in $5 \% \mathrm{CO}_{2}$. Antimicrobial susceptibility testing (AST) was performed using Kirby-Bauer disk diffusion method. AST results were reported for the following antimicrobials: vancomycin, erythromycin, clindamycin, chloramphenicol ceftriaxone, cefotaxime and levofloxacin. Zone sizes were measured and interpreted in accordance with Clinical \& Laboratory Standards Institute (CLSI) guidelines at time of specimen collection. For penicillin, AST was performed by determining the minimum inhibitory concentration (MIC) using the E-test penicillin G strips graduated from 0.016 to 
$256 \mu \mathrm{g} / \mathrm{mL}$ (BioMérieux). Cerebrospinal fluid (CSF) isolates with MIC $\leq 0.06 \mu \mathrm{g} / \mathrm{mL}$ were considered susceptible to penicillin and those with MIC $>0.06 \mu \mathrm{g} / \mathrm{mL}$ were considered non-susceptible. Non-CSF isolates were classified as susceptible, if the MIC was $\leq 2.0 \mu \mathrm{g} / \mathrm{mL}$ and non-susceptible if the MIC was $>2.0 \mu \mathrm{g} / \mathrm{mL}[25,26]$. Starting from September 2017 onwards, the identification of pneumococcal isolates and AST were done using Vitek 2 (BioMérieux) automated system. For all methods, CLSI-recommended quality control checks were done on regular basis.

\subsection{Study Population}

Data on patients and Streptococcus pneumoniae isolates were collected and included the following: age, gender, type of specimen, specimen request date, AST results (susceptible versus non-susceptible) and MIC for penicillin. Multiple isolates collected from the same patient and on the same day and having the same AST results were considered as a single "unique isolate". Unique isolates collected from multiple sites were referred to as "multiple". For isolates that were collected from the same individual on different dates, but having identical AST results, we used the data of the earliest specimen. Isolates from the same individual with different AST results were considered as different unique isolates. Multi-drug resistance (MDR) was defined as non-susceptibility to at least three antimicrobials from the following classes: beta-lactams (penicillin, ceftriaxone or cefotaxime), macrolides (erythromycin), lincosamides (clindamycin) or fluoroquinolones (levofloxacin) [10].

The isolates that were cultured from bloodstream, CSF, peritoneal fluid, pleural fluid or joint fluid were considered as invasive infections, whereas the rest of isolates that were cultured from non-sterile sites were considered non-invasive.

\subsection{Statistical Analysis}

Statistical analysis was conducted through IBM SPSS Statistics 22.0 for Windows. Two-sided Fisher's exact test (FET) was used when appropriate. Statistical significance was considered for $p<0.050$. Temporal trends were analyzed using two-sided linear-by-linear test for association (LBL). Trend analyses were conducted by dividing the study period into quarters, each of which represented 56 months. The 95\% confidence interval (CI) of the prevalence (Wilson score interval, binomial distribution) was calculated using EpiTools epidemiological calculator available online (http://epitools.ausvet.com.au).

\section{Results}

\subsection{Characteristics of Streptococcus pneumoniae Isolates at JUH}

A total of 596 S. pneumoniae isolates were collected at JUH between January 2000 and August 2018 (19 years). Of these isolates, 556 (93.3\%) were unique (i.e., collected from different individuals, or collected from the same individual at different time points with different AST profiles). These unique isolates were collected from 534 different individuals (a single isolate from 515 individuals each, two isolates from 17 individuals, three isolates from a single individual and four isolates from a single individual).

Three-fourths of the total isolates $(n=417,75.0 \%)$ were cultured from five sites as follows: eye $(n=117,21.0 \%)$, bloodstream $(n=93,16.7 \%)$, sputum $(n=81,14.6 \%)$, ear $(n=76,13.7 \%)$ and nasopharynx $(n=50,9.0 \%)$. Data on specimen type could not be retrieved for 42 individuals $(7.6 \%)$; these isolates were neither CSF nor bloodstream isolates, as CSF and bloodstream specimens are always linked to a specific hospital code. A total of 12 unique isolates in multiple specimens from the same individual were found $(2.2 \%)$ as follows: seven from CSF and bloodstream, and five from bloodstream and other specimens (peritoneal fluid $=2$, plural fluid $=1$, joint $=1$ and sputum $=1$ ). A total of 131 isolates (23.6\%) were obtained from invasive infections (bloodstream $=93, \mathrm{CSF}=17$, bloodstream and other sites $=12$, peritoneal fluid $=4$, joint fluid $=3$ and pleural fluid $=2$; Table 1 ) 
A decrease in the prevalence of invasive isolates was found over the study period from $29.5 \%$ in the first quarter to $15.5 \%$ in the fourth quarter $(p<0.001$; LBL, Figure 1$)$.

A $\square$ Total number of unique isolates $\square$ Total number of invasive isolates $\cdots$ Percentage of invasive infections

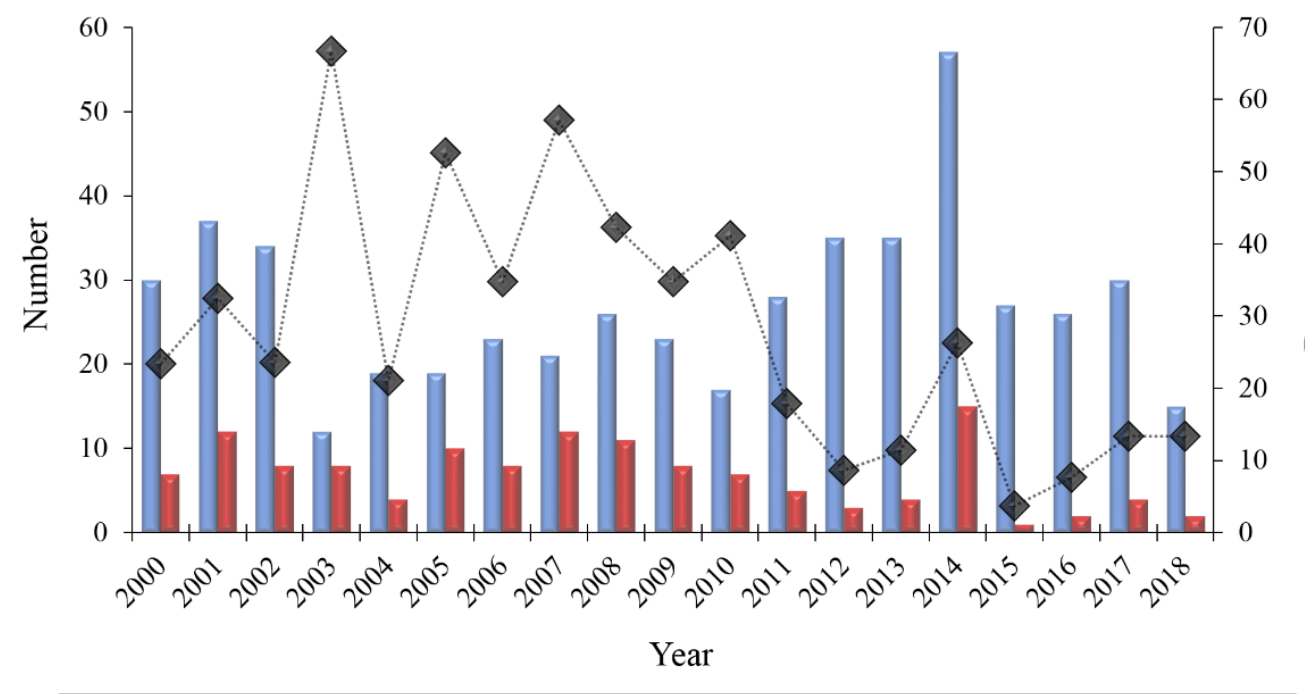

B $\square$ Total number of unique isolates $\square$ Total number of invasive isolates $\bullet$ Percentage of invasive infections

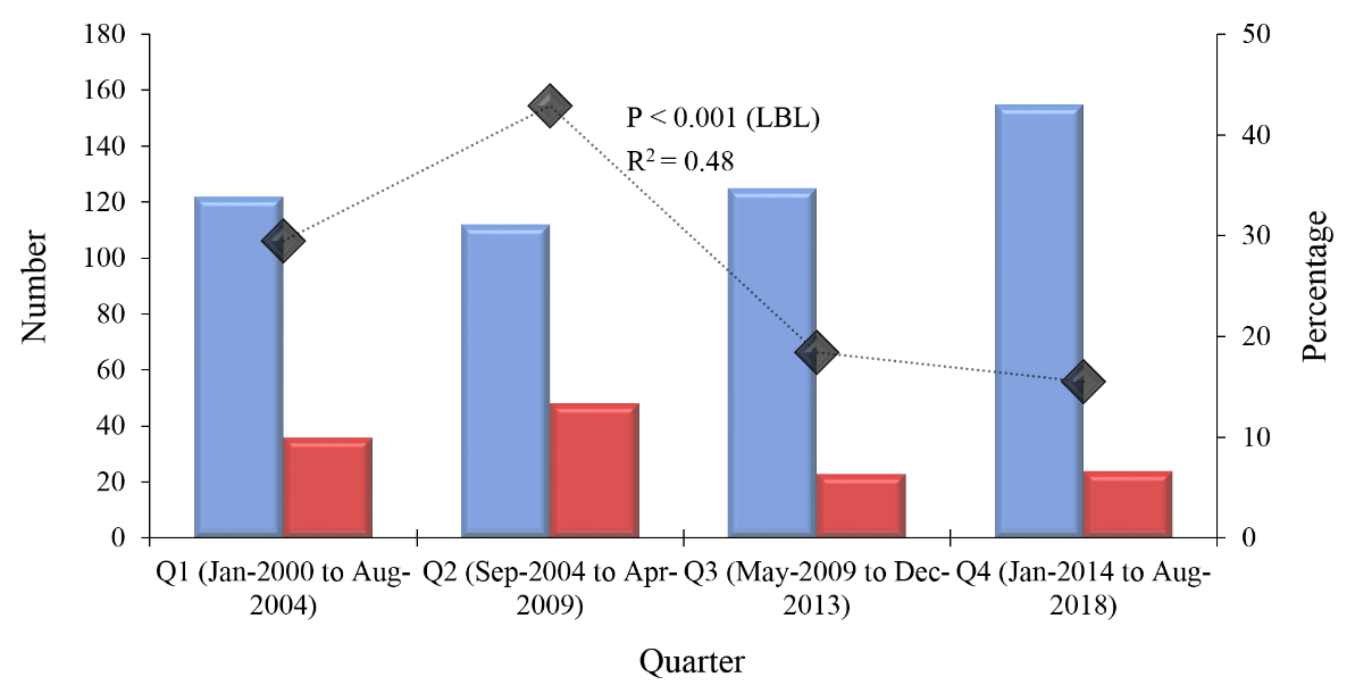

Figure 1. The total number of Streptococcus pneumoniae isolates that were detected at Jordan University Hospital during 2000-2018. (A) The total number of S. pneumoniae isolates stratified per year of isolation are shown in blue and the total number of invasive isolates (isolated from sterile specimens) are shown in red. The line plotted on the secondary axis represents the percentage of invasive isolates per year. (B) The total number of S. pneumoniae isolates stratified per quarter (each of which represented 56 months) are shown in blue and the total number of invasive isolates are shown in red. The line plotted on the secondary axis represents the percentage of invasive isolates per quarter. A decrease in the proportion of invasive isolates was found over the study period $(p<0.001$, linear-by-linear (LBL) test for association LBL). $\mathrm{R}^{2}$ represent the correlation coefficient of the trend line. The total number of isolates plotted in the figure was 514 , as we were not able to retrieve data on specimen type for a total of 42 isolates. 


\subsection{Characteristics of the Study Population}

The total number of unique individuals with pneumococcal isolates was 534. 300 individuals were males $(56.2 \%)$, and the median age of the individuals at time of specimen collection was 10 years (mean: 23, mode: 1, interquartile range [IQR]: 1-43, range: 1-98, data on age were missing for 33 individuals). Nearly two-thirds of the individuals $(n=332)$ were either $\leq 10$ years or $\geq 65$ years. Characteristics of the study population are summarized in (Table 1).

Table 1. Characteristics of the study population and unique Streptococcus pneumoniae isolates that were detected at Jordan University Hospital during 2000-2018.

\begin{tabular}{|c|c|c|}
\hline \multirow{2}{*}{ Characteristic } & \multicolumn{2}{|c|}{ All } \\
\hline & $\mathrm{N}^{1}$ & $\%$ \\
\hline \multicolumn{3}{|l|}{ Gender $^{2}$} \\
\hline Male & 300 & 56.2 \\
\hline Female & 234 & 43.8 \\
\hline \multicolumn{3}{|l|}{ Specimen $^{3}$} \\
\hline Eye & 117 & 21.0 \\
\hline Bloodstream & 93 & 16.7 \\
\hline Sputum & 81 & 14.6 \\
\hline Ear & 76 & 13.7 \\
\hline Nasal & 50 & 9.0 \\
\hline Unknown (non-Blood, non-CSF) ${ }^{4}$ & 42 & 7.6 \\
\hline CSF & 17 & 3.1 \\
\hline Pus & 15 & 2.7 \\
\hline Blood/CSF ${ }^{5}$ & 7 & 1.3 \\
\hline Blood/others 6 & 5 & 0.9 \\
\hline Others $^{7}$ & 53 & 9.5 \\
\hline
\end{tabular}

${ }^{1} \mathrm{~N}$ : Number. ${ }^{2}$ Gender: for unique individuals. ${ }^{3}$ Specimen: for unique isolates, hence, the total number was higher than the total number of unique individuals. ${ }^{4}$ Unknown: information on specimen type could not be retrieved; however, it was possible to confirm that these samples are neither bloodstream nor cerebrospinal fluid (CSF), as they were not assigned with codes of these specimens, ${ }^{5}$ Blood/CSF: dual identical isolates from the same unique individual were collected from both the bloodstream and CSF. ${ }^{6}$ Blood/others: dual identical isolates were collected from the same unique individual with isolates collected from bloodstream and non-CSF specimens. ${ }^{7}$ Others: specimens included: bronchoalveolar lavage fluid $(n=11,2.0 \%)$, throat $(n=10,1.8 \%)$, sinus aspirate $(n=9$, $1.6 \%)$, urine $(n=7,1.3 \%)$, skin $(n=5,0.9 \%)$, peritoneal fluid $(n=4,0.7 \%)$, joint fluid $(n=3,0.5 \%)$, vaginal swab $(n=2,0.4 \%)$ and pleural fluid $(n=2,0.4 \%)$.

\subsection{Antimicrobial Drug resistance Among pneumococcal Isolates in Jordan}

The AST reports were found for at least one antibiotic for a total of 544 unique isolates. The overall susceptibility of S. pneumoniae isolates during the study period to different antimicrobials was as follows: $100 \%$ to vancomycin, $97.7 \%$ to ceftriaxone (95\% CI: 95.5-98.8\%), 97.1\% to cefotaxime (95\% CI: 94.7-98.4\%), 94.9\% to chloramphenicol (95\% CI: 92.7-96.5\%), 89.7\% to penicillin (95\% CI: 86.3-92.3\%), $83.8 \%$ to levofloxacin (95\% CI: 79.7-87.2\%), 67.7\% to clindamycin (95\% CI: $63.6-71.6 \%$ ) and $52.1 \%$ to erythromycin (95\% CI: 47.9-56.3\%). The prevalence of MDR was 8.6\% (95\% CI: 6.4-11.5\%). Details of AMR among pneumococcal isolates in the study are summarized in (Table 2). 
Table 2. Antimicrobial susceptibility testing (AST) results among unique Streptococcus pneumoniae isolates at Jordan University Hospital (2000-2018).

\begin{tabular}{|c|c|c|c|c|c|c|c|c|c|c|c|c|c|c|c|c|}
\hline \multirow{2}{*}{$\begin{array}{c}\text { Antimicrobial } \\
\text { Characteristic }\end{array}$} & \multicolumn{2}{|c|}{ PG } & \multicolumn{2}{|c|}{ CRO } & \multicolumn{2}{|c|}{ CTX } & \multicolumn{2}{|c|}{ CPL } & \multicolumn{2}{|c|}{ LVX } & \multicolumn{2}{|c|}{ CLI } & \multicolumn{2}{|c|}{ ERY } & \multicolumn{2}{|c|}{ MDR $^{2}$} \\
\hline & $\mathrm{S}^{3}$ & $\mathrm{NS}^{4}$ & $S$ & NS & $S$ & NS & S & NS & S & NS & S & NS & S & NS & YES & NO \\
\hline \multicolumn{17}{|c|}{ Gender } \\
\hline Female & 152 & 16 & 142 & 1 & 143 & 1 & 219 & 8 & 136 & 27 & 163 & 67 & 123 & 110 & 20 & 182 \\
\hline \multicolumn{17}{|c|}{ Specimen ${ }^{5}$} \\
\hline Invasive & 82 & 18 & 83 & 3 & 78 & 7 & 127 & 2 & 64 & 6 & 101 & 28 & 80 & 49 & 7 & 108 \\
\hline$<11,>64$ & 238 & 24 & 217 & 5 & 214 & 7 & 315 & 17 & 208 & 39 & 216 & 116 & 161 & 173 & 27 & 271 \\
\hline $11-64$ & 107 & 14 & 102 & 3 & 103 & 3 & 158 & 10 & 92 & 20 & 121 & 49 & 98 & 74 & 12 & 131 \\
\hline \multicolumn{17}{|c|}{ Period $^{7}$} \\
\hline Q1 & 54 & 10 & 30 & 0 & 29 & 1 & 121 & 1 & 1 & 0 & 101 & 21 & 93 & 29 & 1 & 63 \\
\hline Q2 & 62 & 7 & 62 & 6 & 61 & 7 & 103 & 5 & 53 & 0 & 75 & 33 & 61 & 46 & 3 & 91 \\
\hline
\end{tabular}

${ }^{1}$ Antimicrobial: PG: penicillin, CRO: ceftriaxone, CTX: cefotaxime, CPL: chloramphenicol, LVX: levofloxacin, CLI: clindamycin, ERY: erythromycin. All isolates in our study were susceptible to vancomycin. The total number will not add up to 534 for each antimicrobial as AST was not performed for all antimicrobials for all isolates. ${ }^{2}$ MDR: Multi-drug resistance. ${ }^{3}$ S: Susceptible. ${ }^{4}$ NS: Non-susceptible. ${ }^{5}$ Specimen: Invasive specimens include those collected from sterile sites: cerebrospinal fluid, bloodstream, pleural, peritoneal and joint fluid. ${ }^{6}$ Age group: two categories including those younger than 11 years and older than 64 years which was considered a group with higher risk and those between 11 and 64 years as a group with lower risk. Some unique individuals lacked data on age; thus, the total number in this category will be lower than the total number for other categories (gender, specimen and quarter for each antimicrobial). ${ }^{7}$ Period: the study period was divided into four quarters (Q), each of which represents 56 months as follows: Q1 $\rightarrow$ January 2000-August 2004, Q2 $\rightarrow$ September 2004-April 2009, Q3 $\rightarrow$ May 2009-December 2013 and Q4 $\rightarrow$ January 2014-August 2018.

\subsection{Variables that were Associated with Higher Prevalence of AMR}

Upon investigating the variables that could have a possible association with higher prevalence of AMR, we found that resistance to cefotaxime was higher in males than in females $(4.5 \%$ versus $0.7 \%$, $p=0.049$; FET). Cefotaxime resistance was also higher among isolates from invasive infections than non-invasive isolates $(8.2 \%$ versus $1.2 \%, p=0.003$; FET). In addition, isolates from invasive infections had higher prevalence of resistance to penicillin compared to non-invasive isolates $(18.0 \%$ versus $7.8 \%$, $p=0.007 ;$ FET). Conversely, non-invasive isolates had higher prevalence of resistance to clindamycin ( $35.6 \%$ versus $21.7 \%, p=0.003$; FET), to erythromycin $(51.0 \%$ versus $38.0 \%, p=0.011$; FET) and to chloramphenicol $(6.2 \%$ versus $1.6 \%, p=0.037$; FET) in comparison to invasive isolates. All other comparisons yielded statistically non-significant differences. Among the CSF or CSF/bloodstream isolates that had AST results $(n=19)$, the prevalence of non-susceptibility to penicillin was $84.2 \%(95 \%$ CI: $62.4-94.5 \%)$.

\subsection{Temporal Changes of AMR Among pneumococcal Isolates in Jordan}

To investigate the temporal trend of AMR prevalence among the unique pneumococcal isolates, we divided the study period into quarters $(\mathrm{Q})$, each of which represented 56 months: Q1 $\rightarrow$ January 2000-August 2004, Q2 $\rightarrow$ September 2004-April 2009, Q3 $\rightarrow$ May 2009-December 2013 and Q4 $\rightarrow$ January 2014-August 2018. We found an increase in the prevalence of non-susceptibility to clindamycin (from $17.2 \%$ in $\mathrm{Q} 1$ to $40.1 \%$ in Q4, $p<0.001$; LBL), an increase in the prevalence of non-susceptibility to erythromycin (from $23.8 \%$ in Q1 to $58.9 \%$ in Q4, $p<0.001$; LBL), an increase in the prevalence of non-susceptibility to levofloxacin (from $0 \%$ in Q1 to $26.8 \%$ in Q4, $p<0.001$; LBL) and an increase in the prevalence of non-susceptibility to chloramphenicol (from $0.8 \%$ in $\mathrm{Q} 1$ to $7.6 \%$ in $\mathrm{Q} 4, p=0.010$; LBL, Figure 2). In addition, we found an increase in the prevalence MDR from $1.6 \%$ in Q1 to $14.6 \%$ in Q4 ( $p<0.001$; LBL, Figure 2). For penicillin, no significant change was found in the prevalence of non-susceptibility among pneumococcal isolates during the study period ( $p=0.460$; LBL). However, 
we found an incremental increase in the median MIC values over the study period $(0.38 \mu \mathrm{g} / \mathrm{mL}$ in Q1, $0.50 \mu \mathrm{g} / \mathrm{mL}$ in Q2 and $1.0 \mu \mathrm{g} / \mathrm{mL}$ in Q3 and Q4).

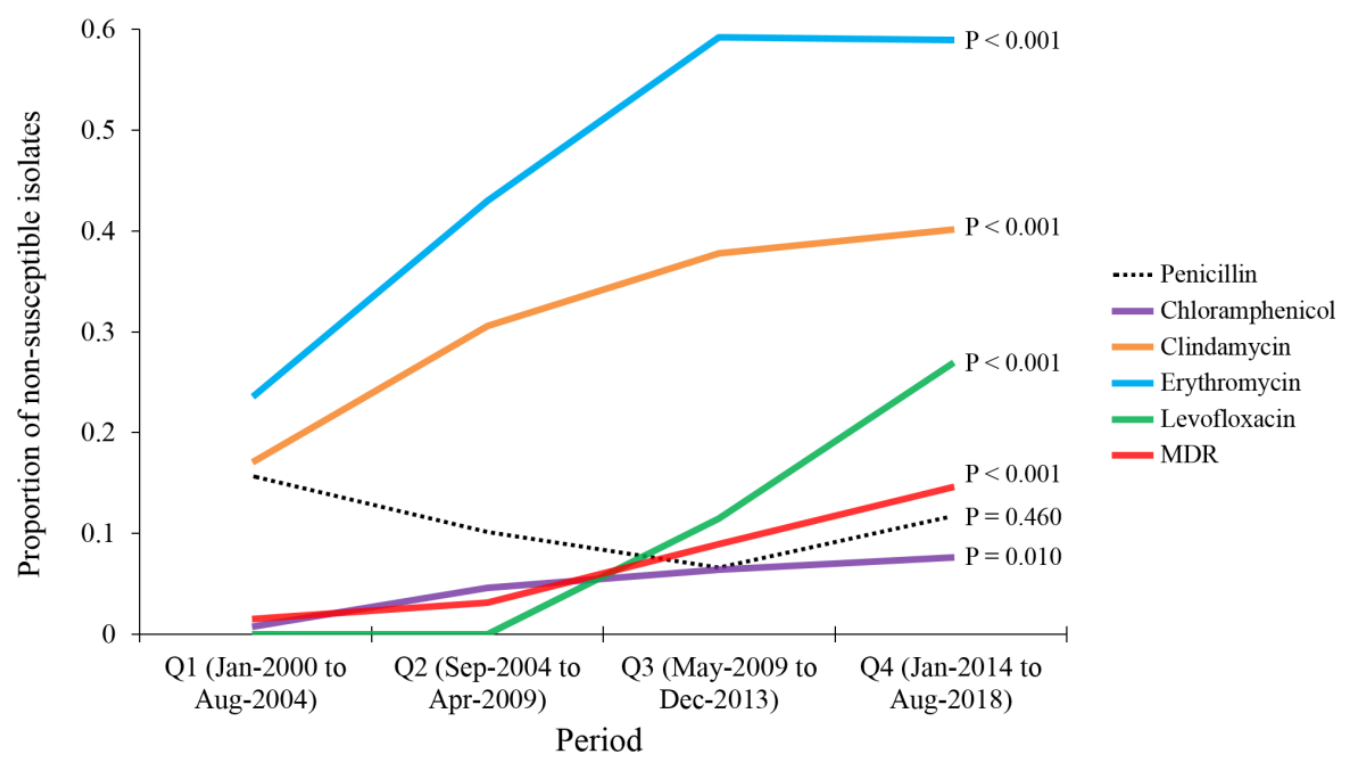

Figure 2. Temporal changes of antimicrobial drug resistance among Streptococcus pneumoniae isolates that were detected at Jordan University Hospital during 2000-2018. Analysis was done by dividing the study period into quarters (Q), each of which represented 56 months: Q1 $\rightarrow$ January 2000-August 2004, Q2 $\rightarrow$ September 2004-April 2009, Q3 $\rightarrow$ May 2009-December 2013 and Q4 $\rightarrow$ January 2014-August 2018. $p$ values were calculated using linear-by-linear test for association. MDR: multi-drug resistance.

\section{Discussion}

The current study represents a large-scale surveillance update on AMR patterns and trends among S. pneumoniae isolates in Jordan over a 19-year period. The study design was based on our attempt to track the long-term changes in the status of antimicrobial drug resistance among pneumococcal isolates in Jordan. Our aim was to assess the changes in resistance patterns that could reflect the local antibiotic prescription practices and treatment guidelines.

In Jordan, pneumococcal conjugate vaccines (PCVs) are not included in the national vaccination schedule, which is related to cost issues (Ministry of Health, Amman, Jordan). However, PCVs have been available since more than a decade for individuals who can afford it. This might explain the significant decrease in the number and proportion of invasive infections over the study period. However, pneumococcal infections in the country continue to represent a huge burden. A recent study from Jordan reported the impact of implementing PCVs and its effect on reducing the carriage rate and AMR among pneumococcal isolates in the individual groups that were studied (infants and children) [27]. Thus, widespread use of PCVs should be considered in Jordan as there is some evidence in literature pointing to possibility of reduction in non-susceptibility to antibiotics following vaccine introduction, even though other study pointed to an opposite effect [28,29].

The main finding of the study was the observation of an increasing prevalence of AMR to drugs that are frequently considered the treatment of choice for pneumococcal diseases [9]. This upward trend in resistance was observed for erythromycin, clindamycin, levofloxacin and chloramphenicol among S. pneumoniae in Jordan over the past 19 years. Similar trend was also reported in past and recent studies from other countries and regions worldwide [18,30-32]. An interesting finding was related to the pattern of levofloxacin non-susceptibility which was non-existent in the first half of the study and reached a rate of $23.5 \%$ in the later half. This finding could be explained by the increased prescription of levofloxacin in the management of pneumococcal disease in Jordan [33]. The emergence of levofloxacin 
non-susceptibility has been reported in Asia and North America from the early 2000s; however, our finding is among the highest reported rates in the world to the best of our knowledge [33-37].

In addition, the proportion of MDR isolates significantly escalated over the study period. Similar pattern was also reported in a previous study from Jordan [24]. However, our study has the advantage of inclusion of a larger sample size from individuals of different age groups with both invasive and non-invasive isolates. This alarming observation of an increasing prevalence of MDR is in line with other reports globally, particularly from the Asian countries [35,38,39]. The observation of an increased prevalence of AMR and MDR necessitates intervention measures, including ongoing AMR surveillance, antibiotic stewardship, consideration of newer drugs with activity against MDR pneumococci and the consideration of introduction of PCVs which might help to reduce resistance [40,41].

Another finding of the study was the stable trend of non-susceptibility to penicillin among S. pneumoniae isolates in Jordan. The overall prevalence of non-susceptibility to penicillin in Jordan during the study period appeared to be low at $10.3 \%$. This finding contrasts the results of the previous and recent reports from the Middle East and North Africa (MENA) region and at the country level $[18,21,22,42,43]$. The accuracy of this finding is related to our adoption of the latest CLSI guidelines for interpretation of the MIC for pneumococcal isolates [25]. The rationale behind using the revised CLSI breakpoints retrospectively for non-CSF isolates is that it gives a better correlation for in vivo susceptibility and a more accurate perspective in epidemiologic surveillance studies [26]. In spite of finding a stable trend of non-susceptibility to penicillin among pneumococcal isolates in this study, two points should be further clarified. First, the study isolates associated with invasive infections showed significantly higher level of resistance to penicillin (particularly for CSF isolates), which discourage the use of penicillin for empiric therapy of such infections. The other point is the incremental increase in the median MIC for penicillin among all pneumococcal isolates in the study. Taken together, the continuous surveillance of penicillin resistance among pneumococci is strongly recommended to revise the local pneumococcal disease treatment guidelines. Contemporary low levels of penicillin non-susceptibility among non-invasive infections together with high levels of resistance among invasive infections were also reported in recent studies from Palestine and Algeria [44,45].

Despite its low prevalence, the alarming observation of non-susceptibility to third-generation cephalosporins (ceftriaxone and cefotaxime), should be taken into consideration particularly among the invasive isolates. A recent study from Taiwan reported a similar finding and suggested treatment options including penicillin, fluoroquinolones or vancomycin to improve the outcome among the patients infected with ceftriaxone-resistant pneumococci [46].

Several limitation points in the study need to be addressed. First, the lack of serotyping and genotyping results, which precluded the assessment of the pneumococcal strains circulating in Jordan. Second, study subjects that were colonized by pneumococci but without any clinical evidence of infection could not be differentiated due to lack of such clinical data. Third, data on community-acquired versus nosocomial infections were missing. Fourth, the interpretation of AST results was based on visual inspection and zone size measurement before September 2017. Thus, a slight difference in AST results might be expected if reporting was done using MICs. Fifth, multiple unique isolates from the same individual can be representative of a single isolate with AMR evolving over time or due to acquisition of a novel pneumococcal strain. Sixth, we cannot rule out sampling bias, based on the findings of frequent isolates from eye specimens and relatively low number of invasive isolates in our study. All these limitations should be considered in the design of future studies tackling the issue of pneumococcal epidemiology in the country.

\section{Conclusions}

The results of the study can be helpful for the improvement of guidelines for empiric therapy of pneumococcal disease in Jordan. The use of penicillin is strongly discouraged in the management of meningitis locally. Ongoing surveillance of pneumococcal AMR and MDR is strongly recommended together with consideration of implementing PCVs in the country. A follow-up prospective 
nationwide study is needed to assess the molecular epidemiology of S. pneumoniae in Jordan using DNA-based methods.

Author Contributions: M.S.: Study conceptualization, supervision, data collection, data analysis and interpretation, writing (original draft preparation), final approval of the manuscript. J.A.: Data collection, data analysis and interpretation, final approval of the manuscript. A.N.: Data collection, data analysis and interpretation, final approval of the manuscript. N.A.A.: Data analysis and interpretation, final approval of the manuscript. A.M.: Study conceptualization, supervision, data analysis and interpretation, final approval of the manuscript. G.Ö.Ş.: Study conceptualization, supervision, data analysis and interpretation, final approval of the manuscript.

Funding: No funding was received for this work.

Conflicts of Interest: The authors declare no conflict of interest.

\section{References}

1. Adegbola, R.A.; DeAntonio, R.; Hill, P.C.; Roca, A.; Usuf, E.; Hoet, B.; Greenwood, B.M. Carriage of Streptococcus pneumoniae and other respiratory bacterial pathogens in low and lower-middle income countries: A systematic review and meta-analysis. PLoS ONE 2014, 9, e103293. [CrossRef] [PubMed]

2. Devine, V.T.; Jefferies, J.M.; Clarke, S.C.; Faust, S.N. Nasopharyngeal Bacterial Carriage in the Conjugate Vaccine Era with a Focus on Pneumococci. J. Immunol. Res. 2015, 2015, 394368. [CrossRef]

3. Torres, A.; Cilloniz, C.; Blasi, F.; Chalmers, J.D.; Gaillat, J.; Dartois, N.; Schmitt, H.J.; Welte, T. Burden of pneumococcal community-acquired pneumonia in adults across Europe: A literature review. Respir. Med. 2018, 137, 6-13. [CrossRef]

4. Mook-Kanamori, B.B.; Geldhoff, M.; van der Poll, T.; van de Beek, D. Pathogenesis and pathophysiology of pneumococcal meningitis. Clin. Microbiol. Rev. 2011, 24, 557-591. [CrossRef] [PubMed]

5. van der Poll, T.; Opal, S.M. Pathogenesis, treatment, and prevention of pneumococcal pneumonia. Lancet 2009, 374, 1543-1556. [CrossRef]

6. O'Brien, K.L.; Wolfson, L.J.; Watt, J.P.; Henkle, E.; Deloria-Knoll, M.; McCall, N.; Lee, E.; Mulholland, K.; Levine, O.S.; Cherian, T.; et al. Burden of disease caused by Streptococcus pneumoniae in children younger than 5 years: Global estimates. Lancet 2009, 374, 893-902. [CrossRef]

7. van de Beek, D.; Cabellos, C.; Dzupova, O.; Esposito, S.; Klein, M.; Kloek, A.T.; Leib, S.L.; Mourvillier, B.; Ostergaard, C.; Pagliano, P.; et al. ESCMID guideline: Diagnosis and treatment of acute bacterial meningitis. Clin. Microbiol. Infect. Off. Publ. Eur. Soc. Clin. Microbiol. Infect. Dis. 2016, 22 (Suppl. 3), S37-S62. [CrossRef] [PubMed]

8. Rivera, A.M.; Boucher, H.W. Current concepts in antimicrobial therapy against select gram-positive organisms: Methicillin-resistant Staphylococcus aureus, penicillin-resistant pneumococci, and vancomycin-resistant enterococci. Mayo Clin. Proc. 2011, 86, 1230-1243. [CrossRef] [PubMed]

9. Aspa, J.; Rajas, O.; de Castro, F.R. Pneumococcal antimicrobial resistance: Therapeutic strategy and management in community-acquired pneumonia. Expert Opin. Pharmacother. 2008, 9, 229-241. [CrossRef]

10. Golden, A.R.; Rosenthal, M.; Fultz, B.; Nichol, K.A.; Adam, H.J.; Gilmour, M.W.; Baxter, M.R.; Hoban, D.J.; Karlowsky, J.A.; Zhanel, G.G. Characterization of MDR and XDR Streptococcus pneumoniae in Canada, 2007-13. J. Antimicrob. Chemother. 2015, 70, 2199-2202. [CrossRef]

11. Linares, J.; Ardanuy, C.; Pallares, R.; Fenoll, A. Changes in antimicrobial resistance, serotypes and genotypes in Streptococcus pneumoniae over a 30-year period. Clin. Microbiol. Infect. Off. Publ. Eur. Soc. Clin. Microbiol. Infect. Dis. 2010, 16, 402-410. [CrossRef] [PubMed]

12. Whitney, C.G.; Farley, M.M.; Hadler, J.; Harrison, L.H.; Lexau, C.; Reingold, A.; Lefkowitz, L.; Cieslak, P.R.; Cetron, M.; Zell, E.R.; et al. Increasing prevalence of multidrug-resistant Streptococcus pneumoniae in the United States. N. Engl. J. Med. 2000, 343, 1917-1924. [CrossRef] [PubMed]

13. Gouveia, E.L.; Reis, J.N.; Flannery, B.; Cordeiro, S.M.; Lima, J.B.; Pinheiro, R.M.; Salgado, K.; Mascarenhas, A.V.; Carvalho, M.G.; Beall, B.W.; et al. Clinical outcome of pneumococcal meningitis during the emergence of pencillin-resistant Streptococcus pneumoniae: An observational study. BMC Infect. Dis. 2011, 11, 323. [CrossRef] [PubMed] 
14. Cherazard, R.; Epstein, M.; Doan, T.L.; Salim, T.; Bharti, S.; Smith, M.A. Antimicrobial Resistant Streptococcus pneumoniae: Prevalence, Mechanisms, and Clinical Implications. Am. J. Ther. 2017, 24, e361-e369. [CrossRef] [PubMed]

15. Johnson, A.P. Surveillance of antibiotic resistance. Philos. Trans. R. Soc. Lond. Ser. B Biol. Sci. 2015, 370, 20140080. [CrossRef] [PubMed]

16. Leekha, S.; Terrell, C.L.; Edson, R.S. General principles of antimicrobial therapy. Mayo Clin. Proc. 2011, 86, 156-167. [CrossRef] [PubMed]

17. Castro-Sanchez, E.; Moore, L.S.; Husson, F.; Holmes, A.H. What are the factors driving antimicrobial resistance? Perspectives from a public event in London, England. BMC Infect. Dis. 2016, 16, 465. [CrossRef]

18. El Moujaber, G.; Osman, M.; Rafei, R.; Dabboussi, F.; Hamze, M. Molecular mechanisms and epidemiology of resistance in Streptococcus pneumoniae in the Middle East region. J. Med. Microbiol. 2017, 66, 847-858. [CrossRef]

19. Mamishi, S.; Moradkhani, S.; Mahmoudi, S.; Hosseinpour-Sadeghi, R.; Pourakbari, B. Penicillin-Resistant trend of Streptococcus pneumoniae in Asia: A systematic review. Iran. J. Microbiol. 2014, 6, 198-210.

20. Verghese, V.P.; Veeraraghavan, B.; Jayaraman, R.; Varghese, R.; Neeravi, A.; Jayaraman, Y.; Thomas, K.; Mehendale, S.M. Increasing incidence of penicillin- and cefotaxime-resistant Streptococcus pneumoniae causing meningitis in India: Time for revision of treatment guidelines? Indian J. Med. Microbiol. 2017, 35, 228-236.

21. Mahafzah, A.; Shehabi, A.A. Antimicrobial susceptibility among clinical isolates of Streptococcus pneumoniae at the Jordan university hospital over an eighteen-year period. Jordan Med. J. 2000, 34, 109-112.

22. Al-Lahham, A.; van der Linden, M. Streptococcus pneumoniae carriage, resistance and serotypes among Jordanian children from Wadi Al Seer District, Jordan. Int. Arabic J. Antimicrob. Agents 2014, 4. [CrossRef]

23. Borg, M.A.; Scicluna, E.; de Kraker, M.; van de Sande-Bruinsma, N.; Tiemersma, E.; Gur, D.; Ben Redjeb, S.; Rasslan, O.; Elnassar, Z.; Benbachir, M.; et al. Antibiotic resistance in the southeastern Mediterranean-Preliminary results from the ARMed project. Euro Surveillance: Bulletin Europeen sur les Maladies Transmissibles = Eur. Commun. Dis. Bull. 2006, 11, 164-167. [CrossRef] [PubMed]

24. Swedan, S.F.; Hayajneh, W.A.; Bshara, G.N. Genotyping and serotyping of macrolide and multidrug resistant Streptococcus pneumoniae isolated from carrier children. Indian J. Med. Microbiol. 2016, 34, 159-165. [CrossRef] [PubMed]

25. CLSI. Performance Standards for Antimicrobial Susceptibility Testing, 26th ed.; CLSI Supplement M100S; Clinical and Laboratory Standards Institute: Wayne, PA, USA, 2016.

26. Weinstein, M.P.; Klugman, K.P.; Jones, R.N. Rationale for revised penicillin susceptibility breakpoints versus Streptococcus pneumoniae: Coping with antimicrobial susceptibility in an era of resistance. Clin. Infect. Dis. Off. Publ. Infect. Dis. Soc. Am. 2009, 48, 1596-1600. [CrossRef]

27. Al-Lahham, A.; Abu Qayyas, J.; van der Linden, M. The Impact of the 7-Valent Pneumococcal Conjugate Vaccine on Nasopharyngeal Carriage of Streptococcus pneumoniae in Infants of Ajlun Governorate in Jordan. Jordan J. Biol. Sci. 2018, 11, 155-162.

28. Hampton, L.M.; Farley, M.M.; Schaffner, W.; Thomas, A.; Reingold, A.; Harrison, L.H.; Lynfield, R.; Bennett, N.M.; Petit, S.; Gershman, K.; et al. Prevention of antibiotic-nonsusceptible Streptococcus pneumoniae with conjugate vaccines. J. Infect. Dis. 2012, 205, 401-411. [CrossRef]

29. Karnezis, T.T.; Smith, A.; Whittier, S.; Haddad, J.; Saiman, L. Antimicrobial resistance among isolates causing invasive pneumococcal disease before and after licensure of heptavalent conjugate pneumococcal vaccine. PLoS ONE 2009, 4, e5965. [CrossRef]

30. van der Linden, M.; Al-Lahham, A.; Haupts, S.; Reinert, R.R. Clonal spread of mef-positive macrolide-resistant Streptococcus pneumoniae isolates causing invasive disease in adults in Germany. Antimicrob. Agents Chemother. 2007, 51, 1830-1834. [CrossRef]

31. Reinert, R.R.; Ringelstein, A.; van der Linden, M.; Cil, M.Y.; Al-Lahham, A.; Schmitz, F.J. Molecular epidemiology of macrolide-resistant Streptococcus pneumoniae isolates in Europe. J. Clin. Microbiol. 2005, 43, 1294-1300. [CrossRef]

32. Anagaw, B.; Gezachew, M.; Biadgelgene, F.; Anagaw, B.; Geleshe, T.; Taddese, B.; Getie, B.; Endris, M.; Mulu, A.; Unakal, C. Antimicrobial susceptibility patterns of Streptococcus pneumoniae over 6 years at Gondar University Hospital, Northwest Ethiopia. Asian Pac. J. Trop. Biomed. 2013, 3, 536-541. [CrossRef] 
33. Bhavnani, S.M.; Hammel, J.P.; Jones, R.N.; Ambrose, P.G. Relationship between increased levofloxacin use and decreased susceptibility of Streptococcus pneumoniae in the United States. Diagn. Microbiol. Infect. Dis. 2005, 51, 31-37. [CrossRef] [PubMed]

34. Hsieh, Y.C.; Chang, L.Y.; Huang, Y.C.; Lin, H.C.; Huang, L.M.; Hsueh, P.R. Circulation of international clones of levofloxacin non-susceptible Streptococcus pneumoniae in Taiwan. Clin. Microbiol. Infect. Off. Publ. Eur. Soc. Clin. Microbiol. Infect. Dis. 2010, 16, 973-978. [CrossRef]

35. Kim, S.H.; Song, J.H.; Chung, D.R.; Thamlikitkul, V.; Yang, Y.; Wang, H.; Lu, M.; So, T.M.; Hsueh, P.R.; Yasin, R.M.; et al. Changing trends in antimicrobial resistance and serotypes of Streptococcus pneumoniae isolates in Asian countries: An Asian Network for Surveillance of Resistant Pathogens (ANSORP) study. Antimicrob. Agents Chemother. 2012, 56, 1418-1426. [CrossRef] [PubMed]

36. Kang, C.I.; Song, J.H. Antimicrobial resistance in Asia: Current epidemiology and clinical implications. Infect. Chemother. 2013, 45, 22-31. [CrossRef] [PubMed]

37. Davidson, R.; Cavalcanti, R.; Brunton, J.L.; Bast, D.J.; de Azavedo, J.C.; Kibsey, P.; Fleming, C.; Low, D.E. Resistance to levofloxacin and failure of treatment of pneumococcal pneumonia. N. Engl. J. Med. 2002, 346, 747-750. [CrossRef] [PubMed]

38. Thummeepak, R.; Leerach, N.; Kunthalert, D.; Tangchaisuriya, U.; Thanwisai, A.; Sitthisak, S. High prevalence of multi-drug resistant Streptococcus pneumoniae among healthy children in Thailand. J. Infect. Public Health 2015, 8, 274-281. [CrossRef]

39. Choi, M.J.; Noh, J.Y.; Cheong, H.J.; Kim, W.J.; Kim, M.J.; Jang, Y.S.; Lee, S.N.; Choi, E.H.; Lee, H.J.; Song, J.Y. Spread of ceftriaxone non-susceptible pneumococci in South Korea: Long-term care facilities as a potential reservoir. PLoS ONE 2019, 14, e0210520. [CrossRef]

40. Ginsburg, A.S.; Klugman, K.P. Vaccination to reduce antimicrobial resistance. Lancet Glob. Health 2017, 5, e1176-e1177. [CrossRef]

41. Van Bambeke, F.; Reinert, R.R.; Appelbaum, P.C.; Tulkens, P.M.; Peetermans, W.E. Multidrug-resistant Streptococcus pneumoniae infections: Current and future therapeutic options. Drugs 2007, 67, 2355-2382. [CrossRef]

42. Al-Yaqoubi, M.M.; Elhag, K.M. Serotype Prevalence and Penicillin-susceptibility of Streptococcus pneumoniae in Oman. Oman Med. J. 2011, 26, 43-47. [CrossRef]

43. Nasereddin, A.; Shtayeh, I.; Ramlawi, A.; Salman, N.; Salem, I.; Abdeen, Z. Streptococcus pneumoniae from Palestinian nasopharyngeal carriers: Serotype distribution and antimicrobial resistance. PLoS ONE 2013, 8, e82047. [CrossRef]

44. Kattan, R.; Abu Rayyan, A.; Zheiman, I.; Idkeidek, S.; Baraghithi, S.; Rishmawi, N.; Turkuman, S.; Abu-Diab, A.; Ghneim, R.; Zoughbi, M.; et al. Serotype distribution and drug resistance in Streptococcus pneumoniae, Palestinian Territories. Emerg. Infect. Dis. 2011, 17, 94-96. [CrossRef]

45. Ziane, H.; Manageiro, V.; Ferreira, E.; Moura, I.B.; Bektache, S.; Tazir, M.; Canica, M. Serotypes and Antibiotic Susceptibility of Streptococcus pneumoniae Isolates from Invasive Pneumococcal Disease and Asymptomatic Carriage in a Pre-vaccination Period, in Algeria. Front. Microbiol. 2016, 7, 803. [CrossRef]

46. Lee, H.Y.; Wu, T.L.; Su, L.H.; Li, H.C.; Janapatla, R.P.; Chen, C.L.; Chiu, C.H. Invasive pneumococcal disease caused by ceftriaxone-resistant Streptococcus pneumoniae in Taiwan. J. Microbiol. Immunol. Infect. $=$ Wei Mian Yu Gan Ran Za Zhi 2018, 51, 500-509. [CrossRef]

(C) 2019 by the authors. Licensee MDPI, Basel, Switzerland. This article is an open access article distributed under the terms and conditions of the Creative Commons Attribution (CC BY) license (http://creativecommons.org/licenses/by/4.0/). 